\title{
EBUS-TBNA hilfreich für die individualisierte Therapie
}

Beim fortgeschrittenen nicht kleinzelligen Bronchialkarzinom

(NSCLC) spielen Subtypisierung sowie Bestimmung des

Mutationsstatus des epidermalen Wachstumsfaktorrezeptors

(EGFR) eine zentrale Rolle für die Therapieentscheidung. Ob

die Zellproben der durch den endobronchialen Ultraschall

unterstützten transbronchialen Nadelaspiration (EBUS-TBNA) für

die Typisierung geeignet sind, haben N. Navani et al. untersucht.

Am J Respir Crit Care Med 2012; 185: 1316-1322

Immer häufiger wird die EBUS-TBNA bei Diagnose und Staging des NSCLC eingesetzt, womit im Prinzip frühzeitig Proben für die Subklassifikation und Genotypisierung zur Verfügung stehen. Ob sie dafür geeignet sind, analysierten die Autoren zwischen 2009 und 2011 anhand von 774 Patienten mit bekanntem oder vermutetem Bronchialkarzinom an 5 Zentren in Großbritannien. Die Zellproben wurden jeweils vom örtlichen Pathologen untersucht, der die morphologische Beurteilung nach der Hämatoxylin-Eosin-Färbung und, wo klinisch indiziert und das Material dazu ausreichte, die Immunfärbung durchführte.

Zum Nachweis eines Plattenepithelkarzinoms wurden Antikörper gegen die Cytokeratine 5/6 und p63 verwendet, daneben auch Antikörper gegen den Schilddrüsentranskriptionsfaktor 1 (TTF1), der bei rund drei Viertel der Adenokarzinome der Lunge exprimiert wird. Der EGFR-Status wurde per DNA-Sequenzierung (PCR) untersucht, wobei der Nachweis von mindestens einer von 29 untersuchten Mutationen als EGFR-positiver Befund gewertet wurde. Alle Patienten wurden mindestens 6 Monate weiter beobachtet und die endgültige Diagnose mit den Befunden der EBUS-TBNA verglichen.

\section{Unspezifizierte Fälle verringern $\nabla$}

Anhand der Zellproben der EBUS-TBNA konnte bei 77\% der Patienten der Subtyp klassifiziert werden (95\%-Konfidenzinterval [KI] 73-80). Insgesamt wiesen 503 Patienten ein NSCLC auf. Bei 101 Patienten konnte nach der EBUS-TBNA bei der Diagnose NSCLC keine weitere Spezifizierung erfolgen (NSCLC-NOS). Das bedeutete für diesen primären Endpunkt der Studie einen nicht näher zu spezifizierendes NSCLC in 23\% der Fälle.

Bei 291 Patienten war zur weiteren Untersuchung eine Immunfärbung indiziert, bei 280 konnte sie auch durchgeführt werden, davon bei 101 Patienten mit NSCLC-NOS. Bei diesen Patienten reduzierte die Immunhistochemie die Rate des NSCLC-NOS um die Hälfte (adjustierte Odds Ratio 0,50; 95\%-KI 0,28-0,82; p=0,016). Eine EGFRMutationsanalyse war bei 107 von 119 Patienten, bei denen dies angefordert wurde, anhand der EBUS-TBNA-Proben möglich. Für die Sensitivität der EBUS-TBNA bei Patienten mit NSCLC ermittelten die Autoren einen Wert von 88\% (95\%-KI 86-91), für den negativen Prädiktivwert 72\% (95\%-KI 66-77) und für die diagnostische Genauigkeit $91 \%$ (95\%-KI 89-93).

\section{Fazit}

Die Studie konnte zeigen, dass die Zellproben der EBUS-TBNA im Routineeinsatz geeignet sind, um die Diagnose eines NSCLC zu sichern und - wo notwendig - eine EGFR-Mutationsanalyse und eine Immunhistochemie durchzuführen. Die Verwendung der Zellproben aus der EBUS-TBNA für die Immunhistochemie kann helfen, die Rate der nicht weiter spezifizierten NSCLC zu senken und so die Chancen für eine individualisierte Therapie zu erhöhen, so die Autoren.

\section{Friederike Klein, München}

\section{Sport senkt Anfallsschwelle}

Sport kann Asthmatikern das Leben deutlich erleichtern und die Symptome der Krankheit lindern. US-amerikanische Forscher des Southwestern Medical Center der Universität in Dallas empfehlen asthmatischen Sportlern, sich vor anspruchsvollen Übungen von einem Arzt untersuchen zu lassen. Die Experten empfehlen Asthmatikern, die regelmäßig trainieren, ihre eigenen Symptome zu beobachten. Auch Unterbrechungen des Schlafs sollten dokumentiert werden.

„Auch die Trainer sollten auf die die Anzeichen und Symptome von Asthma bei den Athleten achten", sagt Rebecca Gruchalla, Leiterin des Bereichs Allergologie und Immunologie des Medical Centers in Dallas. „Mit der sommerlichen Hitze haben die Probleme von Asthmatikern nichts zu tun", erklärt Klaus-Michael Braumann von derDeutschen Gesellschaft für Sportmedizin und Prävention. Im Sommer würden mehr Blüten blühen. Daher sollten Asthmatiker auf einer blühenden Wiese keinen Sport treiben. Generell sei es für Asthmatiker trotzdem gut, Sport zu treiben. „Durch Sport kann die absolute Belastung eines Asthmatikers in einen höheren Bereich verschoben werden", so Braumann. Der Erhalt der Fitness sei eine wichtige Aufgabe der Betroffenen. Denn Asthmatiker bekommen bei maximaler Leistung einen Anfall. Wenn der Betroffene allerdings untrainiert ist, werde er auch beim Treppensteigen einen Anfall bekommen. „Bewegung therapiert das Asthma nicht, aber die Schwelle für einen Anfall wird höher", erläutert Braumann.

Dass sich Spitzensportler oder Athmatiker, die viel Sport treiben, vor der Freiluftsaison mit ihrem Lungen- oder Hautarzt abstimmen, sollte selbstverständlich sein. Dass Asthmatikern aber geraten wird, Sport zu treiben, ist einem Paradigmenwechsel in den vergangenen Jahrzehnten zu verdanken. Inzwischen würden Sportlehrer bereits während ihrer Ausbildung darauf hingewiesen, dass Asthma nicht so gefährlich sei, wie viele vermuten, man aber dennoch auf die betroffenen Kinder achten müsse.

pressetext Nachrichtenagentur $\mathrm{GmbH}$ 